\title{
Gas and Dust in M33
}

\section{J. Braine ${ }^{1}$, P. Gratier ${ }^{1}$, C. Kramer, B. Mookerjea, M. Xilouris and D. Calzetti}

\author{
${ }^{1}$ Laboratoire d'Astrophysique de Bordeaux, Université de Bordeaux, OASU, CNRS/INSU,
} 33271 Floirac, France

\begin{abstract}
We present results from the Herschel and IRAM projects to map M33 in the dust continuum and main emission lines, particularly $\mathrm{C}[\mathrm{II}]$ and CO. The temperature of the cool dust decreases with distance from the center of M33 from $\sim 25 \mathrm{~K}$ to $\sim 13 \mathrm{~K}$. The $\mathrm{CO}$ emission generally follows the dust temperature and the overall dust emission. However, about $1 / 6$ of the molecular clouds are not associated with massive stars, such that about $1 / 6$ th the lifetime of an entity identifiable as a molecular cloud is in a pre-star formation state. These clouds are less CO-bright than those with massive stars. The largest sample of molecular clouds currently available for an external galaxy shows that the cloud CO luminosity function, usually viewed as the cloud $\mathrm{H}_{2}$ mass, steepens with radius such that smaller clouds are more numerous in the outer parts. The observations of the C[II] line with Herschel indicate that the $\mathrm{C}[\mathrm{II}]$ emission traces on-going star formation rather than the neutral gas. This identification will be tested via velocity-resolved Herschel/HIFI C[II] spectra in the near future.
\end{abstract}

Keywords. stars: formation, ISM: molecules, dust - extinction, ISM: evolution, ISM: general, ISM: abundances, galaxies: evolution galaxies: formation, Local Group, infrared: ISM

M33 is a very nearby $(840 \mathrm{kpc})$ spiral galaxy with an optimal inclination - sufficient to study the dynamics of the disk while allowing a clear line of sight (unlike M31). However, there are important differences with our own galaxy that make M33 a very complementary target. M33 has a metallicity $(\mathrm{Z})$ roughly half solar and a mass about a tenth that of the Galaxy. It is blue and gas-rich like early universe objects.

The classical view of a molecular cloud has rather thin layers of ionized then atomic hydrogen (HI) surrounding a predominantly molecular cloud. Since $\mathrm{H}_{2}$ has no rotational transitions, cool $\mathrm{H}_{2}$ is not directly observable and the $\mathrm{CO}$ molecule, the most abundant hetero-nuclear molecule, is used to trace the $\mathrm{H}_{2}$. In low- $\mathrm{Z}$ environments, $\mathrm{CO}$ is probably photo-dissociated deeper into the cloud because of the lower abundance and the lesser shielding against UV radiation by dust and other $\mathrm{CO}$ molecules. This can render the CO emission virtually invisible in extreme low-Z blue objects even if substantial amounts of molecular gas are present. While the model is simple, it is in reality quite difficult to predict the degree to which the CO underestimates the extent and mass of the molecular gas.

M33 is an ideal object to study this question because it represents a first step down in metallicity and shares the global properties of our Galaxy such as a thin spiral disk, a moderate radiation field, and an apparently similar organization of gas into clouds. Because it is so close, the molecular gas can be studied in detail. The Herschel M33 Extended Survey (HerM33ES) key project is providing a huge amount of information on the dust, which is present in the ionized, atomic, and molecular gas components, and on the main gas cooling lines. In particular, the focus on the $\mathrm{C}[\mathrm{II}]$ line at $158 \mu \mathrm{m}$ provides an indication of the photo-dissociation of the $\mathrm{CO}$ (the $\mathrm{C}[\mathrm{II}] / \mathrm{CO}$ ratio should increase with the degree of photodissociation of the $\mathrm{CO}$ in the clouds). The HerM33ES project 


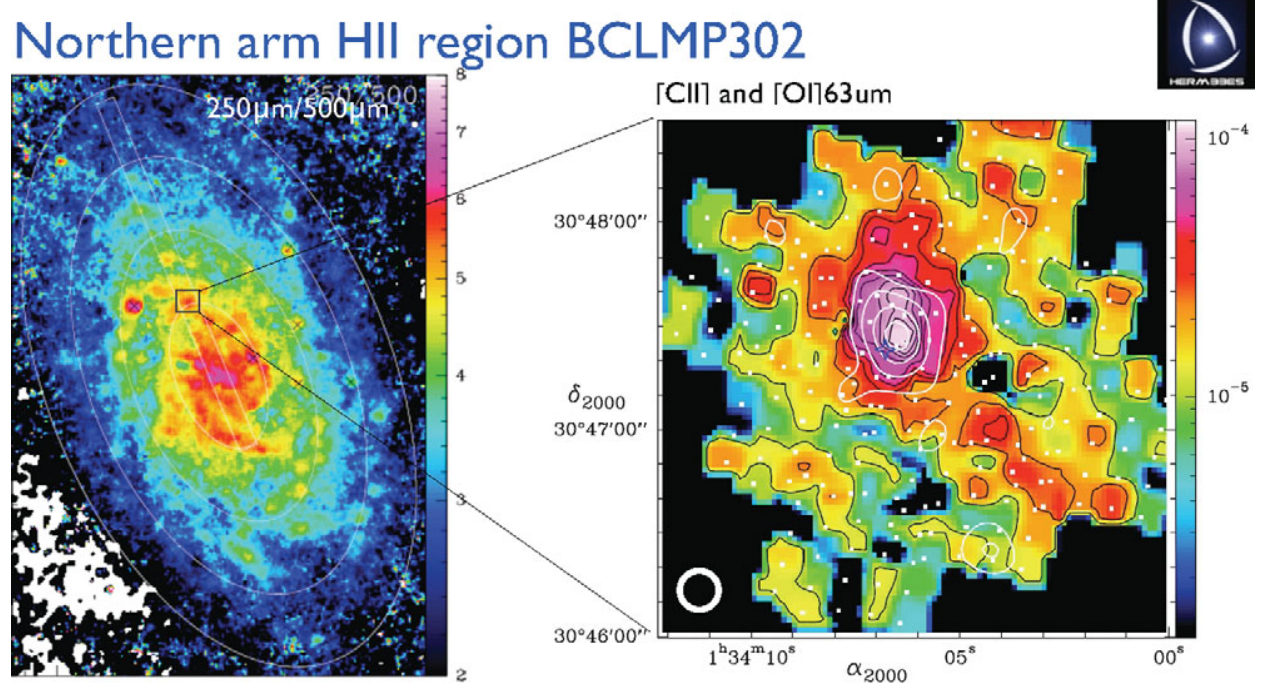

Left: SPIRE $250 \mu \mathrm{m} / 500 \mu \mathrm{m}$ map of $M 33$.

Right: Maps of BCLMP302 showing [CII] emission (color, black contours) and [OI] $(63 \mu \mathrm{m})$ emission (white contours) obtained with PACS at $\sim 12$ " angular resolution ( $50 \mathrm{pc})$.

Star marks peak $\mathrm{H} \alpha$ position observed with $\mathrm{HIFI}$.

Mookerjea, Kramer et al. 2010 in prep.

Figure 1. Left panel indicates the dust temperature (via $250 \mu \mathrm{m} / 500 \mu \mathrm{m}$ flux ratio - red corresponds to $\sim 25 \mathrm{~K}$ and blue to $\sim 13 \mathrm{~K}$ ) and the box indicates the region observed with Herschel/PACS. The PACS C[II] and O[I] results are shown to the right.

will also greatly improve our understanding of the cooling processes of interstellar gas and how they change with the amount of star formation.

Initial results of these projects have been described in Gardan et al. (2007), Gratier et al. (2010a), Kramer et al. (2010), Braine et al. (2010), Verley et al. (2010), and Boquien et al. (2010) with an extension to the Local Group galaxy NGC 6822 in Gratier et al. (2010b). Much of what is presented here is from Gratier (PhD 2010 and in prep.), Mookerjea et al. (submitted), and Xilouris et al. (in prep.).

Figure 1 (left) shows M33 as seen by the Herschel/SPIRE instrument. Although the map presented is actually a ratio of two fluxes, the main features of M33 are reproduced because of the strong color-magnitude link in the Far-IR emission; brighter regions are warmer. The Herschel/PACS C[II](158) and $\mathrm{O}[\mathrm{I}](63)$ line intensities can be seen in the right panel. Both peak at the position of the HII region and the $\mathrm{C}[\mathrm{II}]$ is particularly well correlated with the $\mathrm{H} \alpha$ and $24 \mu \mathrm{m}$ fluxes (Mookerjea et al). The molecular gas (via CO) peaks to the southwest rather than on the HII region.

A Herschel/HIFI spectrum was also taken at the peak in the $\mathrm{H} \alpha$ emission and is shown in Fig. 2. The velocity information provided by HIFI is important in order to understand the origin of the C[II] emission. Particularly interesting is the velocity shift between the $\mathrm{C}[\mathrm{II}]$ and the $\mathrm{CO}$ (and $\mathrm{HI}$ ) which, although small, is significant whereas the CO isotopes are at exactly the same velocity and with a similar line width. The $\mathrm{C}[\mathrm{II}]$ line width is in between that of the $\mathrm{CO}$ and the HI, suggesting it does not come from a warm diffuse component as was proposed by Heiles (1994).

Fig. 3 (left) shows the CO emission (Gratier et al. 2010a) as contours on a plot of the estimated $\mathrm{H}_{2}$ column density (Braine et al. 2010) based on the dust emission as observed 


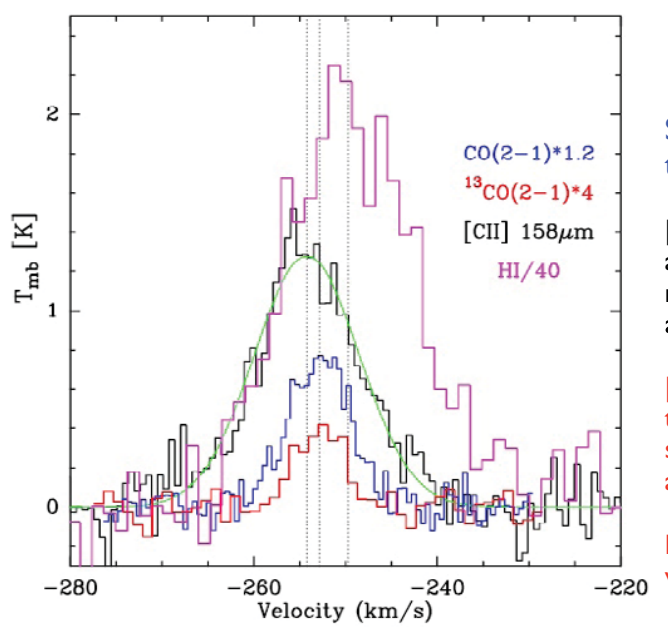

Spectra at the center of the HII region

[CII] integrated intensity agrees within $12.5 \%$ with nearest PACS observation at 3" distance.

[CII] spectrum is broader than $\mathrm{CO}$ (but not $\mathrm{HI}$ ) and shifted relative to the $\mathrm{CO}$ and $\mathrm{HI}$ spectra

Halpha F-P data would be very interesting.

[CII] spectrum taken with HIFI, together with CO IRAM-30m spectra, and a VLA HI spectrum, all at $\sim 12$ " angular resolution.

Mookerjea et al. in prep.

Figure 2. Spectra at the peak of the HII region in $\mathrm{C}[\mathrm{II}], \mathrm{CO},{ }^{13} \mathrm{CO}$, and $\mathrm{HI}$.

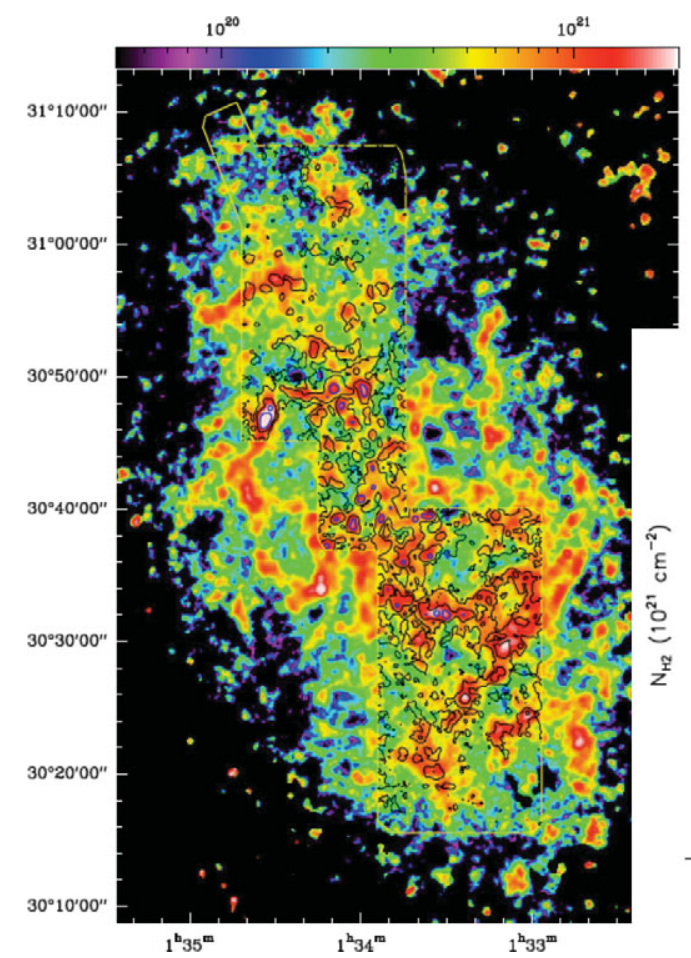

Dust-derived $\mathrm{H} 2$ column density
with $\mathrm{CO}$ contours superposed.

scatter plot of $\mathrm{N}(\mathrm{H} 2)$ vs. Ico with global and inner/outer disk fits $\mathrm{N}\left(\mathrm{H}_{2}\right) / \mathrm{lco}=3,2.2,4.1 \times 10^{20}$

Figure 3. Comparison of $\mathrm{CO}$ and dust-derived $\mathrm{H}_{2}$ column densities.

by SPIRE. The total H column density is determined from the dust temperature, intensity of the Far-IR emission, and a dust cross-section per H-atom and then the HI column density is subtracted. As can be seen, only part of M33, particularly near the major axis, has been observed so far in CO but the CO project should be completed in 2011. Several 


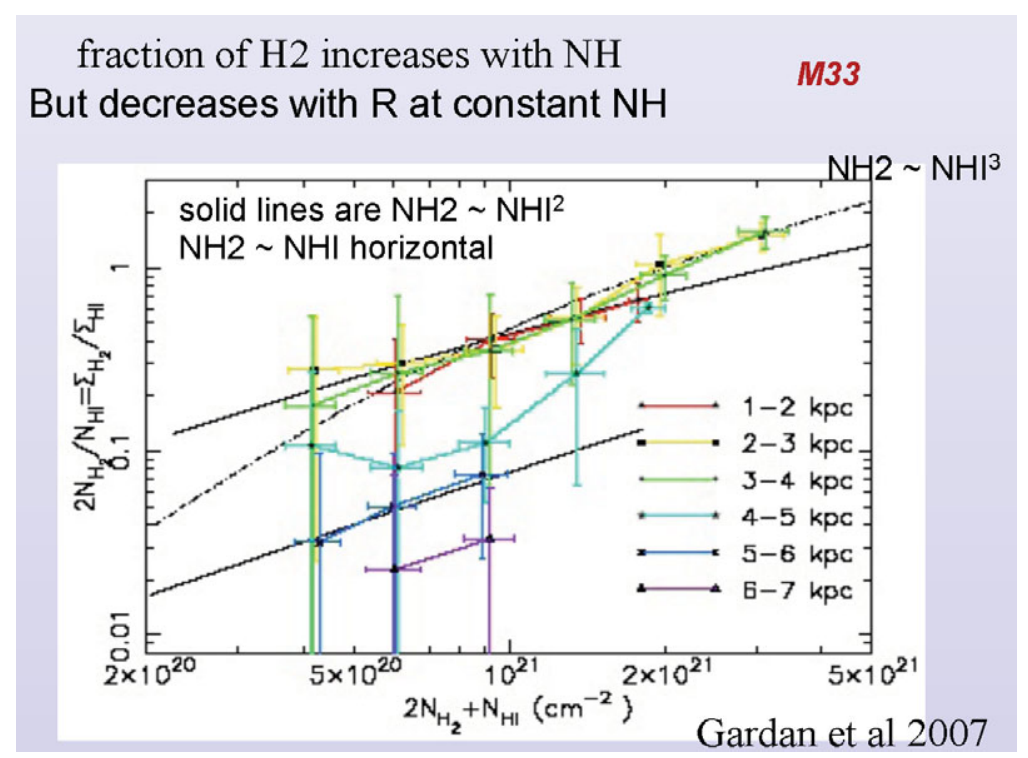

Figure 4. Molecular fraction as a function of total H column density and galactocentric distance.

clouds in the far outer disk of M33 have also been observed in CO at high resolution with the Plateau de Bure interferometer (Braine et al. in prep.). An independent means of comparing the $\mathrm{CO}$ and dust-derived $\mathrm{H}_{2}$ columns is in Fig. 3 (right) where the correlation between the quantities is shown. It appears that the $N\left(\mathrm{H}_{2}\right) / I_{\mathrm{CO}}$ value varies from about 2 to $4 \times 10^{20} \mathrm{H}_{2} /\left(\mathrm{K} \mathrm{km} / \mathrm{s} \mathrm{cm}^{2}\right)$ but that the $\mathrm{CO}$ emission only becomes detectable above column densities of $4 \times 10^{20} \mathrm{~cm}^{-2}$. Fig. 4 shows another interesting result from the $\mathrm{CO}$ emission; while the molecular fraction increases with total $\mathrm{H}$ column density (as expected), for a given total $\mathrm{H}$ column the $\mathrm{H}_{2}$ fraction decreases quickly with radius beyond radii of $4 \mathrm{kpc}$ (details in Gardan et al. 2007).

A cloud identification algorithm was used on the CO cube and 337 GMCs were identified, the largest sample to date in an external galaxy. The CO luminosity function (GMC mass function for a constant $N\left(\mathrm{H}_{2}\right) / I_{\mathrm{CO}}$ ) has a slope close to -2 and if the sample is divided into inner and mid-to-outer disk, then the exponent is about -1.6 near the center increasing to -2.3 for the outer sample, implying a larger fraction of small clouds in the outer disk. The sample was also classified in terms of the star forming activity and $1 / 6$ of the clouds were found not to have star formation as measured by either the $\mathrm{H} \alpha$, FUV, $24 \mu \mathrm{m}$, or $8 \mu \mathrm{m}$ emission (details in Gratier $2010 \mathrm{PhD}$ ).

\section{References}

Boquien, M., et al. 2010, A\&A, 518, L70

Braine, J., et al. 2010, A\& $A$, 518, L69

Gardan, E., et al. 2007, A\&A, 473, 91

Gratier, P., et al. 2010, A\&A, 522, A3

Gratier, P., et al. 2010, A\&A, 512, A68

Heiles, C. 1994, ApJ, 436, 720

Kramer, C., et al. 2010, A\&A, 518, L67

Verley, S., et al. 2010, A\&A, 518, L68 